Bull. Austral. Math. Soc.

$47 \mathrm{H} 09,47 \mathrm{H} 10,65 \mathrm{~J} 15$

VOL. 65 (2002) [109-113]

\title{
ANOTHER CONTROL CONDITION IN AN ITERATIVE METHOD FOR NONEXPANSIVE MAPPINGS
}

\author{
HONG-KUN XU
}

\begin{abstract}
We prove the convergence of an iterative method to a fixed point of a nonexpansive mapping in a uniformly smooth Banach space. We are able to relax one of the control conditions of P.L. Lions (1977).
\end{abstract}

\section{INTRODUCTION}

Let $X$ be a Banach space, $C$ a nonempty closed convex subset of $X$, and $T: C \rightarrow$ $C$ a nonexpansive mapping (that is, $\|T x-T y\| \leqslant\|x-y\|$ for $x, y \in C$ ). (Throughout this article, we always assume that $T$ has a fixed point.) For a fixed $u \in C$ and each $t \in(0,1)$, let $z_{t}$ denote the unique fixed point of the contraction $T_{t}$ given by

$$
T_{t} x:=t u+(1-t) T x, \quad x \in C
$$

Namely: we have

$$
z_{t}=t u+(1-t) T z_{t}
$$

Browder [1] proved that if $X$ is a Hilbert space, then $z_{t}$ converges strongly as $t \rightarrow 0$ to a fixed point of $T$. Reich [4] proved that the same conclusion is true in a uniformly smooth Banach space.

Halpern [2] initiated the study of another (explicit) iterative method:

$$
x_{n+1}=\alpha_{n} u+\left(1-\alpha_{n}\right) T x_{n}, \quad n \geqslant 0
$$

where $x_{0}$ is an arbitrary initial data in $C$ and $\left\{\alpha_{n}\right\}$ is a control sequence in $(0,1)$. He proved the strong convergence of the sequence $\left\{x_{n}\right\}$ if $\left\{\alpha_{n}\right\}$ satisfies certain control conditions, two of which are

(C1) $\lim _{n \rightarrow \infty} \alpha_{n}=0$ and

(C2) $\sum_{n=0}^{\infty} \alpha_{n}=\infty$, or equivalently, $\prod_{n=0}^{\infty}\left(1-\alpha_{n}\right)=0$.

Received 4th June, 2001

The author was supported in part by the National Research Foundation of South Africa.

Copyright Clearance Centre, Inc. Serial-fee code: 0004-9727/02 \$A2.00+0.00. 
Lions[3] improved Halpern's control conditions by showing the strong convergence of $\left\{x_{n}\right\}$ if $\left\{\alpha_{n}\right\}$ satisfies (C1), (C2) and

(C3) $\lim _{n \rightarrow \infty}\left(\alpha_{n+1}-\alpha_{n}\right) /\left(\alpha_{n+1}^{2}\right)=0$.

However, both Halpern's and Lions' controls exclude the natrual choice $\{1 / n\}$ of $\left\{\alpha_{n}\right\}$. This was overcome by Wittmann [7] who proved the strong convergence of $\left\{x_{n}\right\}$ if $\left\{\alpha_{n}\right\}$ satisfies (C1), (C2) and

(C4) $\sum_{n=0}^{\infty}\left|\alpha_{n+1}-\alpha_{n}\right|<\infty$.

Reich [4], Shioji and Takahashi [6] essentially extended Lions' and respectively, Wittman's results to the framework of uniformly smooth Banach spaces. Reich [5] also extended Wittman's result to the class of Banach spaces which are uniformly smooth and have a weakly sequentially continuous duality map. Moreover, the control sequence $\left(\alpha_{n}\right)$ is required to satisfy (C1), (C2) and to be decreasing (and hence (C4) is satisfied).

The purpose of this article is to show that Halpern's result holds in a uniformly smooth Banach space if Lions' control condition (C3) is replaced with the control condition

(C5) $\lim _{n \rightarrow \infty}\left(\alpha_{n+1}-\alpha_{n}\right) /\left(\alpha_{n+1}\right)=0$, or equivalently, $\lim _{n \rightarrow \infty}\left(\alpha_{n}\right) /\left(\alpha_{n+1}\right)=1$.

\section{The Convergence Result}

We need two lemmas.

LEMMA 2.1. Let $\left\{a_{n}\right\}$ be a sequence of nonnegative real numbers satisfying

$$
a_{n+1} \leqslant\left(1-\alpha_{n}\right) a_{n}+\alpha_{n} \beta_{n}, \quad n \geqslant 0
$$

where $\left\{\alpha_{n}\right\}$ is a sequence in $(0,1)$ satisfying the control condition $(\mathrm{C} 2)$ and $\left\{\beta_{n}\right\}$ is a sequence such that $\limsup _{n \rightarrow \infty} \beta_{n} \leqslant 0$. Then $\lim _{n \rightarrow \infty} a_{n}=0$.

Proof: For any $\varepsilon>0$, let $N$ be an integer big enough so that $\beta_{n}<\varepsilon$ for $n \geqslant N$. Repeatedly using (2.1), we obtain, for $n>N$,

$$
a_{n+1} \leqslant\left(\prod_{k=N}^{n}\left(1-\alpha_{k}\right)\right) a_{N}+\left(1-\prod_{k=N}^{n}\left(1-\alpha_{k}\right)\right) \varepsilon .
$$

Condition (C2) then implies that $\limsup a_{n} \leqslant \varepsilon$. This completes the proof.

$$
n \rightarrow \infty
$$

LEMMA 2.2. (Reich [4]) Let $X$ be a uniformly smooth Banach space. Let $C$, $T$, and $\left\{z_{t}\right\}$ be given as in the Introduction. Then the strong limit $\lim _{t \rightarrow 0} z_{t}$ exists and is a fixed point of $T$.

We are now in the position to state and prove the main result of the paper. 
THEOREM 2.3. Let $X$ be a uniformly smooth Banach space, $C$ a closed convex subset of $X$, and $T: C \rightarrow C$ a nonexpansive mapping with a fixed point. Let $u, x_{0} \in C$ be given. Assume $\left\{\alpha_{n}\right\} \subset(0,1)$ satisfies the control conditions (C1), (C2) and (C5). Then the iteration process $\left\{x_{n}\right\}$ defined by (1.3) converges in norm to a fixed point of $T$.

Proof: Let $J$ denote the (normalised) duality map of $X$. Namely,

$$
J(x)=\left\{x^{*} \in X^{*}:\left\langle x, x^{*}\right\rangle=\|x\|^{2}=\left\|x^{*}\right\|^{2}\right\}, \quad x \in X .
$$

It is known that $J$ is the subdifferential of the convex function $\|\cdot\|^{2} / 2: J(x)=$ $\partial 1 / 2\|x\|^{2}, x \in X$. Since $X$ is uniformly smooth, it is also known that $J$ is single-valued and norm-to-norm uniformly continuous on bounded subsets of $X$. The subdifferential inequality

$$
\frac{1}{2}\|v\|^{2} \geqslant \frac{1}{2}\|u\|^{2}+\langle v-u, J(u)\rangle, \quad u, v \in X
$$

implies (and is actually equivalent to) the inequality

$$
\|x+y\|^{2} \leqslant\|x\|^{2}+2\langle y, J(x+y)\rangle, \quad x, y \in X .
$$

For a fixed point $p$ of $T$, it is not hard to see (by induction) that $\left\|x_{n}-p\right\|$ $\leqslant \max \left\{\left\|x_{0}-p\right\|,\|u-p\|\right\}$ for $n \geqslant 0$. In particular, $\left\{x_{n}\right\}$ is bounded and so is $\left\{T x_{n}\right\}$. Hence

$$
\left\|x_{n+1}-T x_{n}\right\|=\alpha_{n}\left\|u-T x_{n}\right\| \rightarrow 0(n \rightarrow \infty) .
$$

It follows from (1.3) that

$$
\begin{aligned}
\left\|x_{n+1}-x_{n}\right\| & =\left\|\left(\alpha_{n+1}-\alpha_{n}\right)\left(u-T x_{n-1}\right)+\left(1-\alpha_{n+1}\right)\left(T x_{n}-T x_{n-1}\right)\right\| \\
& \leqslant\left(1-\alpha_{n+1}\right)\left\|x_{n}-x_{n-1}\right\|+M\left|\alpha_{n+1}-\alpha_{n}\right| \\
& =\left(1-\alpha_{n+1}\right)\left\|x_{n}-x_{n-1}\right\|+\alpha_{n+1} \beta_{n},
\end{aligned}
$$

where $M:=\sup _{n \geqslant 1}\left\|u-T x_{n-1}\right\|$ and $\beta_{n}:=\left(\left|\alpha_{n+1}-\alpha_{n}\right|\right) /\left(\alpha_{n+1}\right) M \rightarrow 0$ by (C5). Hence an application of Lemma 2.1 yields that $\lim _{n \rightarrow \infty}\left\|x_{n+1}-x_{n}\right\|=0$. This together with (2.3) implies that

$$
\lim _{n \rightarrow \infty}\left\|x_{n}-T x_{n}\right\|=0 .
$$

Let $z_{t} \in C(0<t<1)$ be the unique fixed point of the contraction $T_{t}$ given by (1.1). By (1.2) we have

$$
z_{t}-x_{n}=t\left(u-x_{n}\right)+(1-t)\left(T z_{t}-x_{n}\right)
$$


It thus follows from (2.2) that

$$
\begin{aligned}
\left\|z_{t}-x_{n}\right\|^{2} \leqslant & (1-t)^{2}\left\|T z_{t}-x_{n}\right\|^{2}+2 t\left\langle u-x_{n}, J\left(z_{t}-x_{n}\right)\right\rangle \\
\leqslant(1-t)^{2}\left(\left\|T z_{t}-T x_{n}\right\|\right. & \left.+\left\|T x_{n}-x_{n}\right\|\right)^{2} \\
& +2 t\left(\left\|z_{t}-x_{n}\right\|^{2}+\left\langle u-z_{t}, J\left(z_{t}-x_{n}\right)\right\rangle\right) \\
\leqslant\left(1+t^{2}\right)\left\|z_{t}-x_{n}\right\|^{2}+ & \left\|T x_{n}-x_{n}\right\|\left(2\left\|z_{t}-x_{n}\right\|+\left\|T x_{n}-x_{n}\right\|\right) \\
& +2 t\left\langle u-z_{t}, J\left(z_{t}-x_{n}\right)\right\rangle .
\end{aligned}
$$

\section{Hence}

$$
\left\langle u-z_{t}, J\left(x_{n}-z_{t}\right)\right\rangle \leqslant \frac{t}{2}\left\|z_{t}-x_{n}\right\|^{2}+\frac{\left\|T x_{n}-x_{n}\right\|}{2 t}\left(2\left\|z_{t}-x_{n}\right\|+\left\|T x_{n}-x_{n}\right\|\right) .
$$

Taking limsup as $n \rightarrow \infty$ and observing (2.4), we get

$$
\limsup _{n \rightarrow \infty}\left\langle u-z_{t}, J\left(x_{n}-z_{t}\right)\right\rangle \leqslant \limsup _{n \rightarrow \infty} \frac{t}{2}\left\|z_{t}-x_{n}\right\|^{2}
$$

Letting $t \rightarrow 0$, noting the fact that $z_{t} \rightarrow z$ in norm (Lemma 2.2) and the fact that the duality map $J$ is norm-to-norm uniformly continuous on bounded sets of $X$, we get

$$
\limsup _{n \rightarrow \infty}\left\langle u-z, J\left(x_{n}-z\right)\right\rangle \leqslant 0 \text {. }
$$

From (1.3) we can write

$$
x_{n+1}-z=\alpha_{n+1}(u-z)+\left(1-\alpha_{n+1}\right)\left(T x_{n}-z\right) .
$$

Apply the inequality (2.2) to get

$$
\left\|x_{n+1}-z\right\|^{2} \leqslant\left(1-\alpha_{n+1}\right)^{2}\left\|T x_{n}-z\right\|^{2}+2 \alpha_{n+1}\left\langle u-z, J\left(x_{n+1}-z\right)\right\rangle .
$$

Thus

$$
\left\|x_{n+1}-z\right\|^{2} \leqslant\left(1-\alpha_{n+1}\right)\left\|x_{n}-z\right\|^{2}+\alpha_{n+1} \beta_{n}
$$

where $\beta_{n}:=2\left\langle u-z, J\left(x_{n+1}-z\right)\right\rangle$ satisfies $\limsup _{n \rightarrow \infty} b_{n} \leqslant 0$ by (2.5). Apply Lemma 2.1 to (2.6) to conclude that $\lim _{n \rightarrow \infty}\left\|x_{n}-z\right\|=0$; that is, $\left\{x_{n}\right\}$ converges strongly to $z$. 
REMARK 1. It is obvious that condition (C5) is strictly weaker than condition (C3). It is also not hard to see that if $\lim _{n \rightarrow \infty}\left(\alpha_{n}\right) /\left(\alpha_{n+1}\right)$ exists, then condition (C4) implies condition (C5). However, the two conditions are not comparable in general. Indeed, any decreasing sequence $\left\{\alpha_{n}\right\}$ satisfies condition (C4), but not necessary condition (C5); for example, the sequence $\left\{\alpha_{n}\right\}$ given by $\alpha_{n}=e^{-n^{2}}(n \geqslant 1)$ satisfies (C4), but fails to satisfy (C5). On the other hand, the sequence $\left\{\alpha_{n}\right\}$ given by

$$
\alpha_{n}= \begin{cases}\frac{1}{\sqrt{n}}, & \text { if } n \text { is odd } \\ \frac{1}{\sqrt{n}-1}, & \text { if } n \text { is even }\end{cases}
$$

satisfies (C5), but fails to satisfies (C4). We also note that conditions (C3) and (C4) are not comparable either.

REMARK 2. From the proof of Theorem 2.3 one can see that the uniform smoothness assumption of $X$ can be weakened to the uniform Gateaux smoothness of $X$ (equivalently, the duality map $J$ is norm-to-weak * uniformly continuous on bounded subsets of $X$ ) plus the fixed point property for nonexpansive mappings which guarantee the strong convergence of $\left\{z_{t}\right\}$.

\section{REFERENCES}

[1] F.E. Browder, 'Convergence of approximants to fixed points of nonexpansive nonlinear mappings in Banach spaces', Arch. Rational Mech. Anal. 24 (1967), 82-90.

[2] B. Halpern, 'Fixed points of nonexpanding maps', Bull. Amer. Math. Soc. 73 (1967), 957-961.

[3] P.L. Lions, 'Approximation de points fixes de contractions', C.R. Acad. Sci. Paris, Sér. A 284 (1977), 1357-1359.

[4] S. Reich, 'Strong convergence theorems for resolvents of accretive operators in Banach spaces', J. Math. Anal. Appl. 75 (1980), 287-292.

[5] S. Reich, 'Approximating fixed points of nonexpansive mappings', Panamer. Math. J. 4 (1994), 23-28.

[6] S. Shioji and W. Takahashi, 'Strong convergence of approximated sequences for nonexpansive mappings in Banach spaces', Proc. Amer. Math. Soc. 125 (1997), 3641-3645.

[7] R. Wittmann, 'Approximation of fixed points of nonexpansive mappings', Arch. Math. 58 (1992), 486-491.

Department of Mathematics

University of Durban-Westville

Private Bag X54001

Durban 4000

South Africa

e-mail: hkxu@pixie.udw.ac.za 